\title{
A PREEMPTIVE LINK STATE SPANNING TREE SOURCE ROUTING PROTOCOL FOR MOBILE ADHOC NETWORKS
}

\author{
${ }^{1}$ Poonkuzhali Ramadoss, ${ }^{2}$ Sanavullah Mohamed Yakub and ${ }^{1}$ Sabari Annaji \\ ${ }^{1}$ Department of Information Technology, K.S.R. College of Technology, Tiruchengode, Tamilnadu, India \\ ${ }^{2}$ Department of Electrical and Electronics, Annapoorana Engineering College, Salem, Tamilnadu, India
}

Received 2013-08-03; Revised 2013-09-01; Accepted 2013-11-12

\begin{abstract}
Routing protocol is a central issue in Mobile Adhoc Networks (MANET). Several routing protocols have been proposed to deal with the routing issue in MANETs. Routing is a complex task in MANET, if the network is heavily loaded. In this study a new QoS routing protocol is proposed which provides better path selection by avoiding congestion, balancing the load and energy. It minimizes the communication overhead without reducing the network performance. Simulation results show this preemptive link state DFS spanning tree QoS source routing protocol achieves better performance than OLSR and DSDV.
\end{abstract}

Keywords: Mobile Ad Hoc Network (MANET), Quality of Service (QoS), Depth First Search (DFS)

\section{INTRODUCTION}

A Mobile Adhoc Network (MANET) (Boukerch et al., 2011) is a dynamic wireless network without a fixed infrastructure. The nodes communication in MANET is limited within the transmission range. Mostly mobile adhoc networks are used in military communication, in case of earthquake, floods, fire routing protocols in MANET are categorized as proactive and reactive.

Proactive routing protocol maintains regular and up to date routing information about each node in the network by propagating route updation at fixed time intervals throughout the network when there is a change in network topology. Reactive routing protocols establish the route to a destination only when there is a demand for it. Many routing protocols in wireless network are fundamentally derived from two algorithms, Distance Vector (DS) routing and Link State (LS) routing.

In distance vector routing a node provides its neighbours the knowledge of the estimated cost to reach a particular destination. In Link state routing every node provide the cost to its neighbours to all the nodes in the network so every node has the knowledge of the topology of the entire network. For example Adhoc on
Demand Distance Vector (AODV) (Zhang et al., 2009) routing reactive protocol is based on Distance Vector. Destination Sequenced Distance Vector (DSDV) (Khan et al., 2008) is a proactive protocol based on Distance Vector. Optimized Link State Routing (OLSR) (Clausen et al., 2009) is a proactive routing protocol based on Link State.

Another important type of routing protocol is source routing where the entire route is included in the data packet. Source routing protocols provide routing information and also control data forwarding when it is handled by intermediate nodes. The source node selects the entire route, so it avoid congested areas, balances the load and energy consumption among the nodes. Source routing does not require the intermediate nodes have routing capability. The intermediate nodes just forward the data to the next hop neighbor. Also a set of nodes along the path are allowed to help forwarding data which are included as an ordered list in the data packets.

Link state routing algorithms gives each node the knowledge of the complete topology of the network, it could be utilized for source routing as in (Rozner et al., 2013). In this study, a preemptive link state DFS spanning tree QoS source routing protocol for MANET

Corresponding Author: Poonkuzhali Ramadoss, Department of Information Technology, K.S.R. College of Technology, Tiruchengode, Tamilnadu, India 
is proposed to complement OLSR and DSDV. The primary goal is to minimize the communication overhead without reducing the network performance. This is proved by the performance evaluation and comparison with OLSR and DSDV using simulation experiments in ns-2.

The rest of the paper is organized as follows. Section 2 reviews the literature survey on source routing. Section 3 describes the design of preemptive link state DFS spanning tree. Section 4 gives the implementation details of preemptive link state DFS spanning tree QoS source routing protocol. Section 5 shows the performance evaluation and the results of the experiments. Section 6 concludes the paper.

\section{LITERATURE SURVEY}

Optimized Link State Routing (OLSR) is a table driven routing protocol and naturally preserves the specialties of proactive protocols. It uses an optimized link state scheme, which is based on the technique multipoint relaying (MRP) to diffuse topology information. The operation of OLSR consists of a necessary core function and a set of auxiliary functions which could be optimally utilized according to specific scenarios.

In DSDV every node in the network maintains a routing table in which all the possible destinations within the network as well as the number of hops to reach each destination are recorded. Each route entry is marked with a sequence number which is assigned by the destination. Nodes periodically transmit routing table updates throughout the network in order to maintain table consistency.

DSR (Duncan and Eddie, 2012) is an on demand adhoc network routing protocol composed of two parts: Route Discovery and Route maintenance. A distinct feature of DSR is that it uses route cache strategy to reduce routing messages in the whole network. Every host holds a route cache table which updates periodically to get the later information about the network. DSR has since been extended to meet different objectives. Flow ID is used to identify the route in the network as in (Tang and Long, 2008). Explicit signals are used to inform the upstream nodes when detecting broken links to better utilize the cached routes in (Husieen et al., 2011).

In STAR (Garcia-Luna-Aceves and Spohn, 1999) every node maintains a tree structure for a network and adopts a tree update strategy that is neither proactive nor reactive. Instead it uses a lazy approach where update messages will only be transmitted when the local tree structure is considered sufficiently inferior to the original optimum. In this all the tree updates are performed differently and the link states are time stamped.

In this study working and performance of a new preemptive link state DFS spanning tree QoS source routing protocol is presented. It is built on the similar idea of spanning tree as in STAR. But it does not timestamp as in STAR. Only the topological information is used for tree updates to reduce the communication overhead. It maintains a depth first spanning tree at each node to provide responsive data transportation services. To strike the balance between efficient and robust network operations a full dump and differential updates are utilized. Source routing is used to forward the data rather than IP forwarding as in STAR.

\section{DESIGN OF PREEMPTIVE LINK STATE DFS SPANNING TREE}

In this Preemptive QoS Source Routing Protocol (PSRP) every node has a depth first spanning tree (West, 1996) of the entire network. Nodes periodically broadcast tree structure information to the best of its knowledge. A node can expand its scope of knowledge about the network based on the information it has been collected from its neighbours. Then this knowledge is exchanged among all the neighboring nodes in the next iteration.

The operation of PSRP is iterative and distributed among all the nodes in the network.

At the beginning the node "a" is aware of the existence of its neighbours by listening to their hello packets. There it is able to construct DFS Spanning Tree (DFST) rooted at itself within $\mathrm{N}_{1}$ (a). In each subsequent iterations nodes exchange their spanning tree with their neighbours. In the $i^{\text {th }}$ iteration $(i=1,2,3,4, \ldots)$ node " $a$ " receives a broadcast message from each of its neighbours. The message from the neighbour $b_{k}$ contains the DFST of $\mathrm{N}_{\mathrm{i}-1}\left[\mathrm{~b}_{\mathrm{k}}\right]$ rooted at $\mathrm{b}_{\mathrm{k}}$.

This tree is denoted as $\mathrm{D}_{b_{k}}^{\mathrm{i}-1}$. Node "a" calculates its own DFST based on $\mathrm{D}_{\mathrm{b}_{\mathrm{k}}}^{\mathrm{i}-1}$ and $\mathrm{D}_{\mathrm{a}}^{\mathrm{i}-1}$.

This is done by $\mathrm{D}_{a}^{\mathrm{i}-1} \bigcup_{k=1}^{N(a)} \mathrm{D}_{b_{k}}^{i-1}$ calculating a DFST of the union of all these trees.

If the tree contains any cycle the following procedure is used to break it.

Consider the example of node "a" and its neighbor $b_{k}$ in Fig. 1:

- If the cycle is odd the newly added edge connects two vertices of same distance, so remove this new edge 


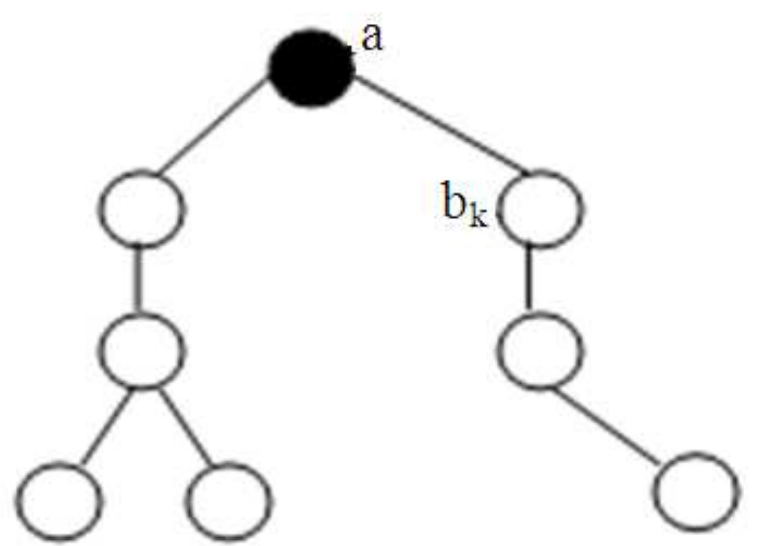

Fig. 1. Adding edge

- If the cycle is even the node will have two paths, so keep the original edge in the path and remove the new edge

After incorporating all the edges the DFST of $\mathrm{N}_{\mathrm{i}}(\mathrm{a})$ tree $\mathrm{T}_{\mathrm{a}} \mathrm{i}$ is constructed. At the end the node " $\mathrm{a}$ " broadcasts $\mathrm{T}_{\mathrm{a}} \mathrm{i}$ to all of its neighbours.

Let the network diameter be $\mathrm{D}$ hops. After D iterations each node in the network has constructed a DFST of the entire network rooted at itself.

\section{IMPLEMENTATION OF PREEMPTIVE LINK STATE DFS SPANNING TREE QOS SOURCE ROUTING PROTOCOL}

\subsection{Reduction of Communication Overhead}

The DFST information stored at a node is to be broadcasted to its neighbours. To do that first the general rooted tree is converted into binary tree of same size of nodes. Then the binary tree is serialized using a bit sequence of 34 bits. The binary tree is scanned layer by layer. First the IP address of the node is included in the sequence. In addition two more bits are appended to indicate if it has a left and/or right child Fig. 2.

The above tree is represented as a11b11c10d11e00f10g00h00i00. Once in t cycles each node is allowed to broadcast the full tree structure in a message. But in every cycle, a node broadcasts "update message" to depict the changes in its locally stored DFST. Here $t$ is set as 3 to make a balance.

After a node receives a update message from a neighbour it extracts the edges and use them to reconstruct the new DFST. This requires each node to store an old copy of the DFST. This requires each node to store an old copy of the DFST for each of its neighbours.

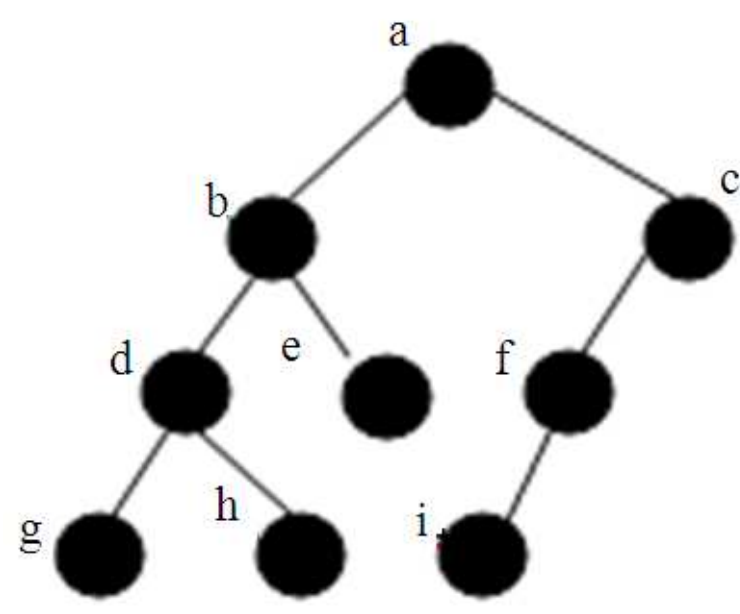

Fig. 2. Binary tree

\subsection{Finding Unreachable Nodes}

Before transmitting the periodical route information the source node first verifies if it is connected to all its neighbours. That is when the source node does not receive the periodic broadcast message from its neighbour in the previous cycle, it considers the link to the neighbour is lost. If it is so then it will follow the repair procedure as follows:

- Trimming the branches from the tree by removing all the broken links from its DFST

- The source node then repairs its own tree by incorporating the stored copies of the DFSTs of its neighbours. By receiving the route message from a neighbor the source node update the stored set of unreachable nodes for the neighbor as well

\subsection{Building the Route}

After the construction of DFST when the source node has received a route update from its neighbour it incorporates this information to the destination as follows:

- If the neighbour node indicates that a node which is unreachable is reachable again, the augmentation of source's spanning tree is similar to growing the tree

- If the neighbor node indicates that the node has a shorter distance than the source is aware of, the source node adopts this neighbor as next hop to reach the destination unless it forms a loop 


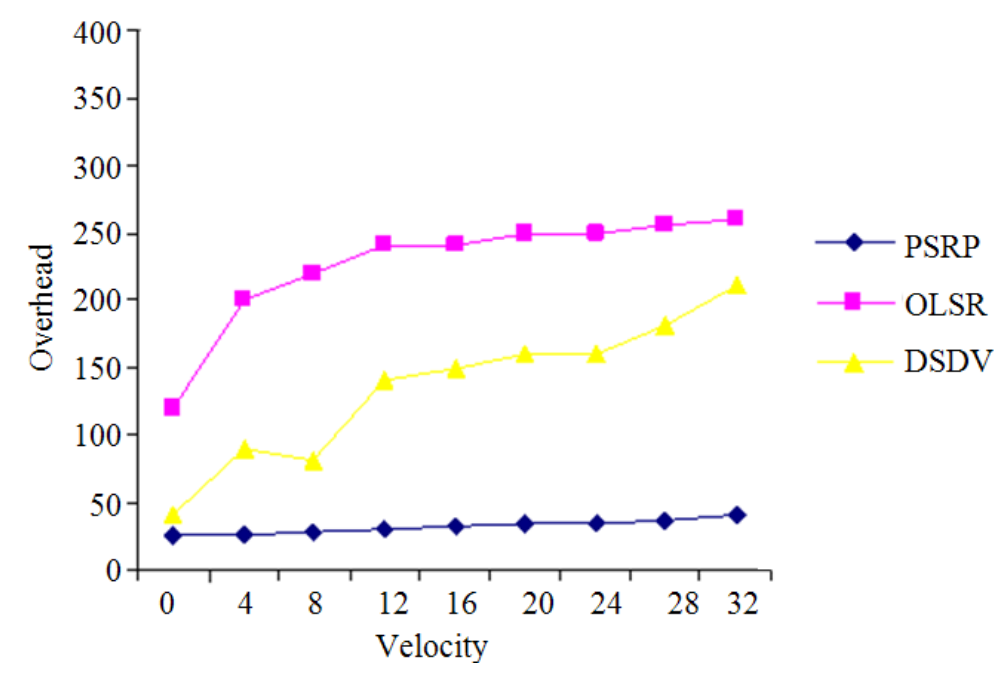

Fig. 3. Overhead vs node velocity

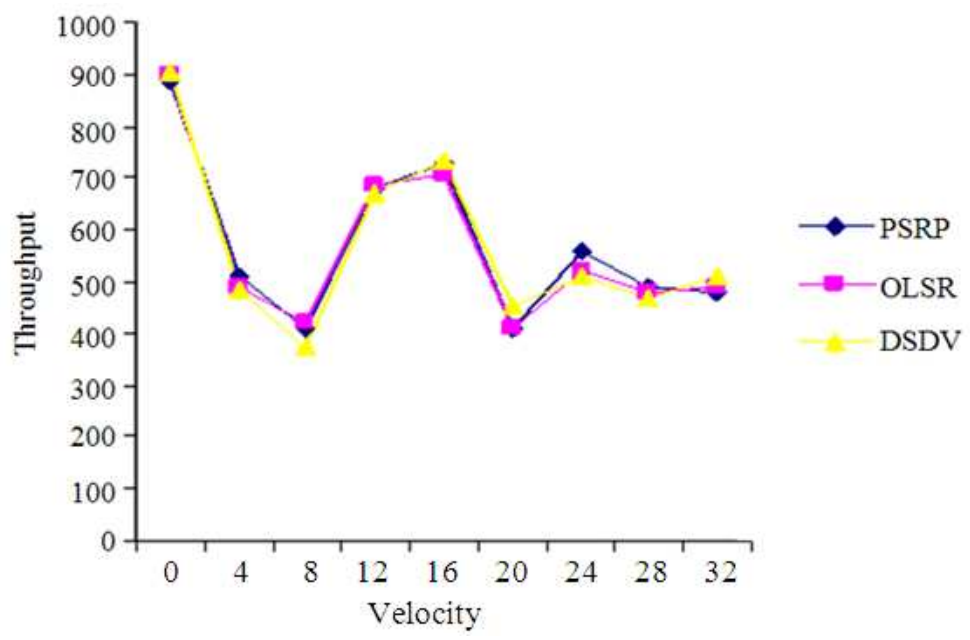

Fig. 4. Throughput vs node velocity

- If the neighbor node indicates longer distance instead and if the source node considers it as the next hop to reach the destination then it queries the stored trees of all its neighbours. If any neighbour has a better route to destinations source nodes DFST is modified using that neighbour as the next hop to reach destination

\section{PERFORMANCE EVALUATION}

The performance of PSRP is measured by using network simulator ns-2. The results are compared with OLSR and DSDV with varying node mobility rates. It is observed that the overhead of PSRP is only a fraction of OLSR and DSDV also it achieves similar performance in transporting TCP data flows when compared with the other two protocols.

\subsection{Experiment Settings}

The random waypoint model is adopted to model node mobility to generate simulation scenarios. In this model each node moves towards a series of target positions. The rate of velocity for each move is uniformly selected from $\left[0, V_{\max }\right]$. Once it has reached a target position it may pause for a specific amount of time before moving towards the next position. 


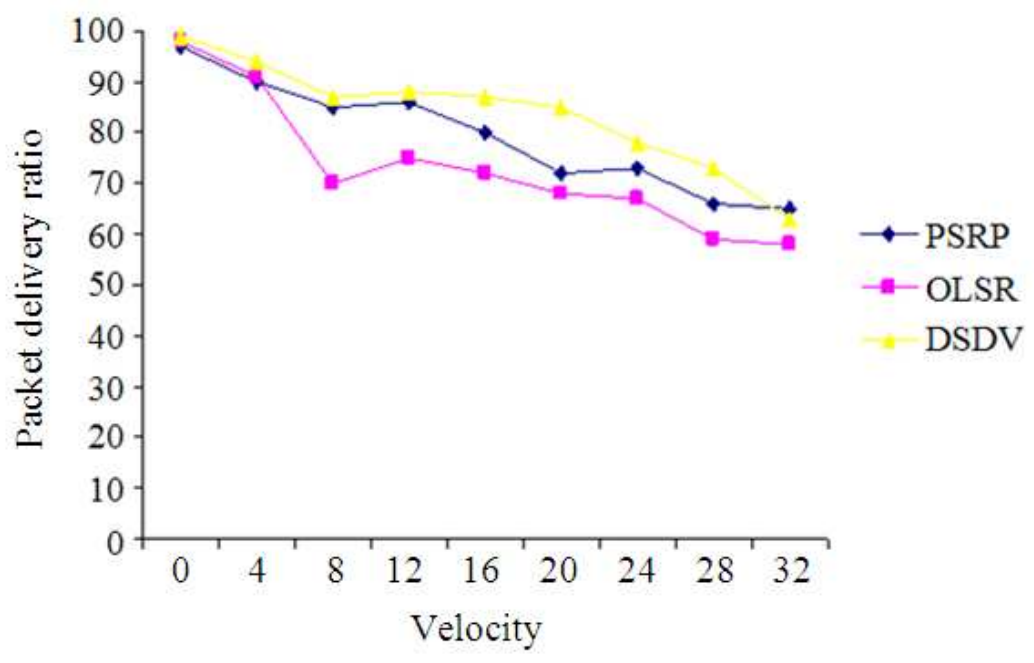

Fig. 5. Packet delivery ratio vs nodevelocity

\subsection{Performance Analysis}

The scenario is with 50 nodes deployed in $1100 \times 1100$ sq.m square area with velocity set to $0,4,8,12, \ldots 32(\mathrm{~m} / \mathrm{s})$. Each node has a density of around 7 neighbours.

The routing overhead of PSRP, OLSR and DSDV are measured by varying the node velocity.

It is observed in Fig. 3 that as the velocity increases the overhead of all the protocols comes down. PSRP has only a very small fraction of the overhead of OLSR and DSDV. In contrast both OLSR and DSDV's overhead increases with node mobility.

The throughput performance of all the three protocols is little affected in the scenario where node velocity is varying as shown in Fig. 4. The performance of PSRP, OLSR and DSDV are similar with PSRP leading the pack in most cases. Throughput is not affected by the different rates of velocity. When $\mathrm{V}_{\max }=0$ all protocols yield a high throughput of $900 \mathrm{kbps}$.

The packet delivery ratio of PSRP, OLSR and DSDV is studied. Again their relative performance is similar as shown in Fig. 5. Packet delivery ratio of PSRP is always $70 \%$ even when $\mathrm{V}_{\max }=32$. Packet delivery ratio drops gradually as the node velocity increases.

\section{CONCLUSION}

The protocol provides source routing using DFS spanning tree routing in MANET. To provide responsive data transfer capability in MANETs, a preemptive source routing protocol is preferred. Though OLSR employs the array of optimization techniques, its overhead remains high in presence of the constrained communication resources in MANETs. Thus PSRP is designed which provides nodes with the cost of network structure information for source routing at a communication overhead similar or even less than a distance vector routing protocol. In PSRP nodes maintain and exchange DFSTs periodically. The full dump message which contains the entire spanning tree is of the size $O(|n|)$ which is less frequently broad cast than a compact differential updates. PSRP yields the same transportation capability as the more expensive protocol like OLSR and DSDV.

\section{REFERENCES}

Boukerch, A., B. Turgut, N. Aydin, M.Z. Ahmad and L. Boloni et al., 2011. Routing protocols in ad hoc networks: A survey. Comput. Networks, 55: 30323080. DOI: 10.1016/j.comnet.2011.05.010

Clausen, T., C. Dearlove and P. Jacquet, 2009. The optimized link state routing protocol. IETF Internet Draft Version 2.

Duncan, N. and B.T. Eddie, 2012. An energy-efficient dynamic source routing protocol for mobile ad hoc networks. Int. J. Comput. ICT Res., 6: 23-32.

Garcia-Luna-Aceves, J.J. and M. Spohn, 1999. Sourcetree routing in wireless networks. Proceedings of the 7th Annual International Conference on Network Protocols, Oct. 31-Nov. 3, IEEE Xplore Press, Toronto, Canada, pp: 273-282. DOI: 10.1109/ICNP.1999.801950 
Husieen, N.A., O.B. Ghazali, S. Hassan and M.M. Kadhum, 2011. Route cache update mechanisms in DSR protocol-A survey. Proceedings of the International Conference on Information and Network Technology, (INT' 11), IACSIT Press, Singapore, pp: 136-141.

Khan, K.U.R., A.V. Reddy, R.U. Zaman, K.A. Reddy and T.S. Harsha, 2008. An Efficient dsdv routing protocol for wireless mobile ad hoc networks and its performance comparison. Proceedings of the 2nd UKSIM European Symposium on Computer Modeling and Simulation, Sept. 8-10, IEEE Xplore Press, Liverpool, pp: 506-511. DOI: 10.1109/EMS.2008.11

Rozner, E., M.K. Han, L. Qiu and Y. Zhang, 2013. Model-driven optimization of opportunistic routing. IEEE/ACM Trans. Network., 21: 594-609. DOI: 10.1109/TNET.2012.2205701
Tang, S.K. and D. Long, 2008. Multiple disjoint paths routing using implicit source routes in wireless ad hoc networks. Proceedings of the International MultiConference of Engineers and Computer Scientists, Mar. 19-21, Hong Kong, pp: 1047-1047.

West, D.B., 1996. Introduction to Graph Theory. 1st Edn, Prentice Hall, Upper Saddle River, NJ, ISBN10: 0132278286, pp: 512.

Zhang, L., H. Sun, Q. Sun, X.Y. Hu and C.J. Guo, 2009. Improvement of routing protocols AODV in wireless ad hoc network. Proceedings of the WRI World Congress on Software Engineering, May 1921, IEEE Xplore Press, Xiamen, pp: 470-472. DOI: 10.1109/WCSE.2009.396 